\title{
Onset of thermomagnetic convection around a vertically oriented hot-wire in ferrofluid
}

\author{
Ashkan Vatani ${ }^{* 1,2}$, Peter Lloyd Woodfield ${ }^{1}$, Nam-Trung Nguyen ${ }^{2}$, Dzung Viet Dao ${ }^{1,2}$ \\ ${ }^{1}$ School of Engineering, Griffith University, Gold Coast Campus, Gold Coast, QLD 4222, Australia \\ ${ }^{2}$ Queensland Micro- and Nanotechnology Centre, Brisbane, QLD 4111, Australia \\ *ashkan.vatani@griffithuni.edu.au
}

\begin{abstract}
The onset of thermomagnetic convection in ferrofluid in a vertical transient hot-wire cell is analytically and experimentally investigated by studying the temperature rise of an electrically-heated wire. During the initial stage of heating, the temperature rise is found to correspond well to that predicted by conduction only. For high electrical current densities, the initial heating stage is followed by a sudden change in the slope of the temperature rise with respect to time as a result of the onset of thermomagnetic convection cooling. The observed onset of thermomagnetic convection was then compared to that of natural convection of deionized water. For the first time, the critical time corresponding to the onset of thermomagnetic convection around an electrically-heated wire is characterized and non-dimensionalized as a critical Fourier number $\left(F o_{c}\right)$. We propose an equation for $\mathrm{Fo}_{c}$ as a function of a magnetic Rayleigh number to predict the time for the onset of thermomagnetic convection. We observed that thermomagnetic convection in ferrofluid occurs earlier than natural convection in non-magnetic fluids for similar experimental conditions. The onset of thermomagnetic convection is dependent on the current supplied to the wire. The findings have important implications for cooling of high-power electronics using ferrofluids and for measuring thermal properties of ferrofluids.
\end{abstract}

Keywords: Onset, Thermomagnetic convection, free convection, ferrofluid, electrically heated-wire

\section{Introduction}

Ferrofluid is a unique type of nanofluid with many potential and existing applications in biomedicine, point-of care diagnostics and heat transfer [1-3]. Because of its potential, research related to ferrofluid has attracted great attention during the past decade [4-6]. The differences between ferrofluid and conventional nanofluids are related to its magnetic properties, which when exposed to a magnetic field with a non-uniform temperature distribution result in a unique type of convection called "thermomagnetic convection" [7-9]. Thermomagnetic convection occurs due to variation of magnetic susceptibility and hence changes in the magnetic body-force distribution as a result of temperature gradients in the fluid. Unlike natural convection, time-dependent thermomagnetic convection around a heated wire has been given very little attention in the literature [10].

The onset of natural convection has been investigated in numerous researches [11-14]. The onset of convection is defined as the point where the heat flux characteristic changes from conduction to convection. For natural convection, heat transfer from a vertical cylinder and the transition from the conduction regime to natural convection have been well documented [15-19]. The onset of natural convection effects in a transient hot-wire system was experimentally studied by Ro et al. [17] for air, carbon tetrachloride $\left(\mathrm{CCl}_{4}\right)$ and n-Hexane $\left(n-\mathrm{C}_{6} \mathrm{H}_{14}\right)$. The authors determined the dimensionless upper limiting time for the measurement of the thermal conductivity of fluids as a function of modified 
Rayleigh number $\left(R a^{*}=N u \cdot R a\right)$. Zhang et al. [19] studied the natural convection of various fluids in transient short-hot-wire cells with different lengths of wire. The team correlated the nondimensionalized critical time corresponding to the onset of natural convection $\left(F o_{c}\right)$ as a function of both Rayleigh number and wire aspect ratio $\left(\frac{L}{D}\right)$. Later, Woodfield et al. [18] numerically simulated the natural convection for the short-hot-wire in Hydrogen and Argon, where the results were in good agreement with the correlation proposed by Zhang et al [19].

The effect of magnetic field on natural convection has also been investigated by a number of researchers for electrically-conducting fluids in studies on magneto-hydrodynamics (MHD) (e.g. [13, 20, 21]). Increasing the Rayleigh number and Lewis number accelerates the onset of natural convection in a nanofluid layer (e.g. [11, 21, 22]), while increasing the magnetic Chandrasekar number [23] delays the phenomenon. Sarkar et al [14] developed a comprehensive theoretical model to investigate the effect of a transverse magnetic field on the buoyancy-driven convection heat transfer of electrically-conducting nanofluids in an infinitely long channel.

The effect of a magnetic field on thermomagnetic convection and other types of body-force driven convection in magnetic and electrically-conducting fluids has been studied in [24-29]. Engler and Odenbach [30] experimentally studied the onset of thermomagnetic convection in a horizontal ferrofluid layer by measuring the critical combined (magnetic and thermal) Rayleigh number ( $R a+$ $R a_{m}$ ) for a range of frequencies of driving force. In their experiment the time-modulation of driving force was supplied by sinusoidal variations of temperature gradient over a fluid gap. They observed that for low frequencies the $R a_{\text {comb }}$ had a steep increase, corresponding to delayed onset of convection. The $R a_{\text {comb }}$ appeared to decrease smoothly after a certain value of frequency. In another experiment, the same researchers [31] observed that for the case of a constant magnetic field applied, the critical temperature for the onset of convection is decreased with increasing magnetic field intensity. Tynjala and Ritvanen [32], in a numerical study of the thermomagnetic convection in an annulus between two concentric cylinders, found the limiting values for the onset of pure buoyancy-driven convection and pure thermomagnetic convection in absence of gravity. Engler et al [33] investigated a horizontal layer of ferrofluid subjected to vertical homogeneous magnetic field and temperature gradient. They observed that the critical temperature difference for the onset of thermomagnetic convection was shifted to higher values in the presence of a magnetic field. They also reported that the increased viscosity of ferrofluid as a result of the magnetoviscous effect has a stabilizing effect on thermomagnetic convection (delays the onset of convection). Therefore, they concluded that the onset of magnetic convection, in addition to magnetic field strength and temperature gradient, can be highly dependent on the thermal properties of the ferrofluid.

The general conditions for the onset of various instabilities under the influence of a magnetic field have been investigated in a number of studies. Lange [34] used a linear stability analysis to determine the critical external induction for the onset of thermomagnetic convection of magnetic fluid in a cylindrical geometry. Shliomis et al [35] studied the onset of thermomagnetic convection in a ferrofluid with inhomogeneous distribution of magnetic particles as a result of either magnetophoresis or thermophoresis. Nanjundappa et al [36] used a weakly nonlinear stability analysis to find the criteria for the onset of thermomagnetic convection in a ferrofluid saturating a porous layer in the presence of a uniform vertical magnetic field and under the effect of Coriolis force. Sekar et al [37] analysed the role of the Soret effect on the stability of a multi-component fluid in an anisotropic porous medium heated from below and salted from above using Brinkman model. Nanjundappa et al [38] investigated the effect of temperature-dependent viscosity on the onset of Benard-Marangoni 
ferroconvection in a horizontal layer of ferrofluid. Gear et al [39] experimentally determined the critical magnetic field below which no magnetically-driven surface deformations occur for the ferrofluid. Saravanan and Yamaguchi [40] using a combination of analytical and numerical methods calculated the critical conditions for the onset of convection in a magnetic-fluid-filled differentially heated porous layer placed in a zero-gravity environment.

Knowledge of the time for the onset of convection effects is important for transient methods for measuring fluid thermal conductivity. Studies done on the onset of natural convection have largely been motivated by the need to avoid convection effects when measuring thermal conductivity of fluids $[15,41]$. In the transient hotwire method for measuring thermal conductivity, the temperature rise of the hotwire due to Joule heating is measured at any instant and plotted against the logarithm of time. Then the slope of the linear region of the plot, where heat conduction is the dominating means of heat dissipation from the wire to the fluid, is used to calculate the thermal conductivity of the fluid [42]. The main feature of this technique is its short measurement time. It is of particular importance that the data necessary to measure the thermal conductivity should be collected before the onset of convection [43]. Therefore, determining the time corresponding to onset of convection effects is valuable for measuring thermal conductivity and thermal diffusivity of fluids.

Although the critical conditions for the onset of instability under influence of magnetic field have been proposed in a number of investigations, the time for the onset of thermomagnetic convection around a heated wire in ferrofluid has never been studied. Also, a survey over the literature suggests that in almost all studies on thermomagnetic convection, the source of heat flux has invariably been separated from the source of magnetic field which has been either a magnet or a line dipole placed outside of the ferrofluid. In the present study, for the first time, the time for the onset of thermomagnetic convection in ferrofluid under influence of a non-uniform magnetic field induced around a vertical hotwire is analytically correlated and experimentally validated and compared to the time for the onset of free convection of deionized water (DIW).

\section{Theoretical model}

The induced magnetic field around the current-carrying wire immersed in an electrically nonconducting ferrofluid (no electromagnetic free current induced in the flow) is characterised by Maxwell's relations as:

$$
\begin{gathered}
\vec{B}=\mu_{0}(\vec{H}+\vec{M})=\mu_{0}(1+\chi) \vec{H} \\
\vec{M}=\chi \vec{H}
\end{gathered}
$$

A constant current applied to the wire results in Joule heating and imposes a temperature gradient on the sample liquid. This temperature gradient results in a non-uniform magnetic susceptibility of the ferrofluid. Temperature dependence of the magnetic susceptibility has been expressed in the Langevin equation:

$$
\chi=\frac{n\left\langle m^{2}\right\rangle}{3 k_{B} T}
$$


where $n$ is the numerical concentration of ferroparticles and $\left\langle m^{2}\right\rangle$ is the mean squared ferroparticle magnetic moment. Eq. (3) shows that as the temperature increases the susceptibility (and hence the magnetic body force on the fluid) decreases.

Finlayson [44] explained that, similar to thermogravitational convection, the non-uniform magnetic susceptibility distribution in the fluid results in a non-uniform magnetic body force which makes the colder fluid with higher susceptibility flow toward the larger magnetic field strength region (i.e. toward the wire in this study). This phenomenon is called the thermomagnetic convection. The magnetic body force applied to an electrically nonconducting incompressible ferrofluid is given by:

$$
\vec{f}=\nabla(\vec{M} \cdot \vec{B})
$$

The Kelvin body force in cylindrical coordinates can be derived by substituting appropriate expressions for $\vec{M}$ and $\vec{B}$ corresponding to the magnetic field around a single wire and $\chi=\Delta \chi+\chi_{0}$, where $\chi_{0}$ is the magnetic susceptibility at a reference temperature, into Eq.(4) to obtain:

$$
f=\frac{-\mu_{0}\left(1+\chi_{0}\right) \chi_{0} I^{2}}{2 \pi^{2} r^{3}}+\frac{-\mu_{0}\left(1+2 \chi_{0}\right) \Delta \chi I^{2}}{2 \pi^{2} r^{3}}
$$

The first term on the RHS of Eq. (5) is the hydrostatic term (i.e. unaffected by changes in the velocity field), and the second term is the dynamic driving force arising from changes in the magnetic susceptibility due to the temperature field as shown in Eq. (3). Applying the Maclaurin series expansion and neglecting higher order terms, the magnetic susceptibility change due to temperature gradient is given by:

$$
\Delta \chi \approx-\chi_{0} \frac{\Delta T}{T_{0}}
$$

Shifting the hydrostatic component of the magnetic body force into the pressure gradient term by redefining the pressure as $P^{*}=P-\frac{\mu_{0}\left(1+\chi_{0}\right) \chi_{0} I^{2}}{2 \pi^{2} r^{3}}$, the Navier-Stokes equations can be expressed as:

$$
\rho \frac{D \vec{V}}{D t}=-\nabla P^{*}+\eta \nabla^{2} \vec{V}+\frac{\mu_{0}\left(1+2 \chi_{0}\right) \chi_{0} I^{2}}{2 \pi^{2} r^{3} T_{0}} \Delta T \hat{r}+\rho \vec{g}
$$

Where $\rho \vec{g}$ represents the gravitational buoyancy force and the third term on the right hand side is the magnetic body force.

A magnetic Rayleigh number was derived from Eq. (7) by Vatani et al [10] for this geometry, using a similar procedure to Incropera's derivation for the Rayleigh number of free convection [45]:

$$
R a_{m}=\frac{\mu_{0}\left(1+2 \chi_{0}\right) \chi_{0} I^{2} \Delta T r_{c e l l}{ }^{3}}{2 \pi^{2} \alpha \eta r_{w i r e} T_{0}}
$$


This magnetic Rayleigh number expresses the thermomagnetic force to viscous force ratio multiplied by the Prandtl number, characterising the thermomagnetic convection heat transfer. By analogy with transient natural convection, the Fourier number representing the time to the onset of thermomagnetic convection effects is expected to be a function of the magnetic Rayleigh number from Eq. (8), the Nusselt number and the length-to-diameter ratio for the wire.

\section{Measurement method}

The measurement set up used in this experiment is essentially the same as the one used in the transient hot-wire method to measure the temperature rise of the wire. The measurement cell designed for this experiment includes a circular channel, $50 \mathrm{~mm}$ in length and $5 \mathrm{~mm}$ in diameter, embedded in an acrylic block, with a 50- $\mu$ m diameter copper wire passing through the centre of the channel and soldered to thin copper tapes used to seal the two ends of the channel (Figure 1). In this experiment a power supply (KEITHLEY 2200-20-5, US) was used to provide a constant current to the microwire and consequently generate heat via joule heating which dissipated into the fluid. The power supply is connected to the microwire via lead wires. Joule heating causes a rise in the temperature of the wire over time. To measure the temperature rise, first the voltage across the hot wire is measured using a multimeter (KEITHLEY 2000, US) and with the aid of a designed LabView ${ }^{\mathrm{TM}}$ program data is recorded for voltage vs. time. Then the resistance of the wire at each instance is calculate from $R=\frac{V}{I}$. And finally the resistance is converted into temperature of the wire as $T=\frac{\left(\frac{R}{R_{0}}-1\right)}{T C R}$ where $R_{0}$ is the resistance of the wire at $0^{\circ} \mathrm{C}$ and TCR is the temperature coefficient of resistance of the wire. The set up was calibrated to find the TCR of the wire as explained in [46]. The measurement was placed in a temperature controlled bath (ISOTECH RS422, UK) to ensure uniform temperature around the cell.

After measuring the temperature change with time, the heat transfer coefficient $h$ is calculated from its definition as the proportionality coefficient in Newton's law of cooling as,

$$
h=\frac{q}{A\left(T_{\text {wire }}-T_{i}\right)}
$$

where $q=R I^{2}$ is the generated power, $A$ is the surface area of the wire, $T_{\text {wire }}$ is the temperature at the surface of the wire and $T_{i}$ is the temperature of the bath.

The Nusselt number of the flow is calculated as,

$$
N u_{L}=\frac{h L}{k}
$$

where $L=0.05 \mathrm{~m}$ is the length of the wire, and $k$ is the thermal conductivity of the ferrofluid predicted by the Corcione's correlation [47] at any given temperature. Fourier number representing the dimensionless time is calculated as: 


$$
F_{O}=\frac{\alpha t}{r_{\text {wire }}^{2}}
$$

Where wire radius is used as characteristic length.

A ferrofluid provided by Ferrotec ${ }^{\mathrm{TM}}$ (Singapore) with $2 \%$ nanoparticle volume concentration and average nanoparticle diameter of $10 \mathrm{~nm}$ was used as the test fluid. $F o_{c}$ for the onset of natural convection and thermomagnetic convection is determined at different temperatures in a range of $10{ }^{\circ} \mathrm{C}$ to $40{ }^{\circ} \mathrm{C}$ with four different currents of $1.5 \mathrm{~A}, 2 \mathrm{~A}, 2.5 \mathrm{~A}$ and $3 \mathrm{~A}$ being supplied to the wire.

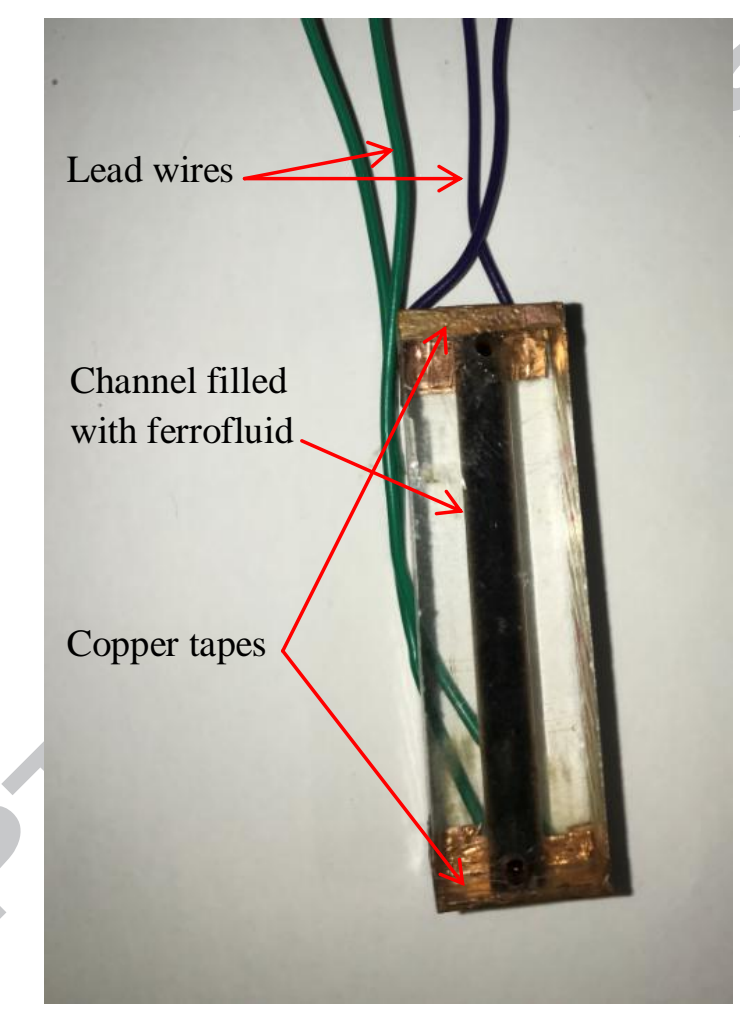

Figure 1 Measurement cell used in the experiment filled with ferrofluid.

\section{Sensitivity analysis}

To investigate the relative importance of various parameters on measured outputs, an uncertainty analysis was performed as listed in Table 1. All calculations for propagation and combination were done based on standard uncertainties. The uncertainty in the length of the cell measured for 5 different cells was $\pm 1 \mathrm{~mm}$. The uncertainty in the measured temperature of the wire owing to the uncertainty in the calibrated TCR of the wire is around $2 \%$. The uncertainty in the supplied DC current and measured voltage is $0.05 \%$ and $0.001 \%$, respectively (according to device manual), which has been neglected in this analysis. The initial bath temperature was measured to have up to $0.5^{\circ} \mathrm{C}$ uncertainty. The radius of the 
measurement cell was assumed to have 5\% uncertainty and other parameters considered in this analysis were assumed to have $1 \%$ uncertainty. The results in Table 1 shows that $F o$ is not greatly affected by the uncertainty in the measurement, while $N u$ is affected by the uncertainty in temperature readings, length of the wire and radius of the wire. The magnetic Rayleigh number is the most sensitive to uncertainty in the measured inputs.

Table 1 Uncertainty analysis

\begin{tabular}{cccccc}
\hline Input & Uncertainty & $\mathrm{Ra}(\%)$ & $\mathrm{Ra}_{\mathrm{m}}(\%)$ & $\mathrm{Nu}(\%)$ & $\mathrm{Fo}(\%)$ \\
\hline$\beta$ & $1 \%$ & 1 & - & 1 & - \\
$k$ & $1 \%$ & - & - & - & - \\
$\alpha$ & $1 \%$ & - & 1 & - & 1 \\
$\eta$ & $1 \%$ & - & 1 & - & - \\
$v$ & $1 \%$ & 1 & - & 2.2 & - \\
$\Delta T$ & $2 \%$ & 2.2 & 3.3 & 0.63 & - \\
$T_{0}$ & $0.5^{\circ} \mathrm{C}$ & 0.71 & 1.58 & 1.95 & - \\
$L$ & $2 \%$ & 6.1 & - & - & - \\
$r_{\text {cell }}$ & $5 \%$ & - & 11.7 & 3.2 & 1 \\
Combined & & 6.7 & 12.3 & & \\
\hline
\end{tabular}

\section{Results and discussion}

Figure 2 shows the temperature rise of the wire for a 5 second heating period. In the initial linear region of the plot, heat conduction is the dominating means of heat transfer. However, after a given time span the temperature rise deviates from linearity. The time at which temperature rise deviates $5 \%$ from the slope of conduction curve is defined as the critical time corresponding to the onset of convection. This definition is consistent with the criterion used in Zhang et al's study on natural convection [19]. For the example shown in Figure 2 the critical time for the onset of natural convection in deionized water (DIW) is 1.4 seconds while for the case of ferrofluid onset of thermomagnetic convection occurs at 0.47 seconds. Non-dimensionalizing these times gives critical Fourier numbers $\left(F o_{c}\right)$ of 339 for the natural convection case and 116 for the ferrofluid case. 


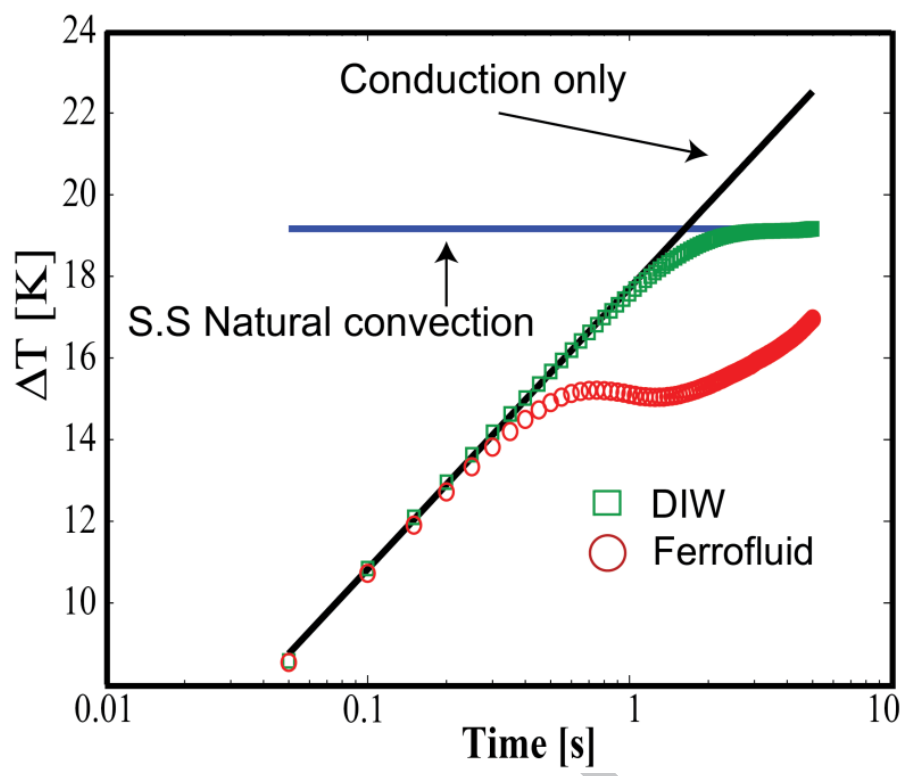

Figure 2 Temperature rise of the wire at $40^{\circ} \mathrm{C}$ bath temperature when $1.5 \mathrm{~A}$ current is supplied.

To validate the experimental results of the present study, $F o_{c}$ for the onset of free convection in DIW is compared with results reported in the literature. It is shown in Figure 3 that present experimental results are in good agreement with the correlation proposed by Zhang et al [19] for estimating the critical time for the onset of natural convection in deionized water as a function of aspect ratio (L/D), Rayleigh number (Ra) and Nusselt number (Nu). In Zhang et al's correlation, $R a^{*}=R a$. $N u$ is the modified Rayleigh number and $\frac{L}{D}$ term has been added to consider the effect of the wire aspect ratio.

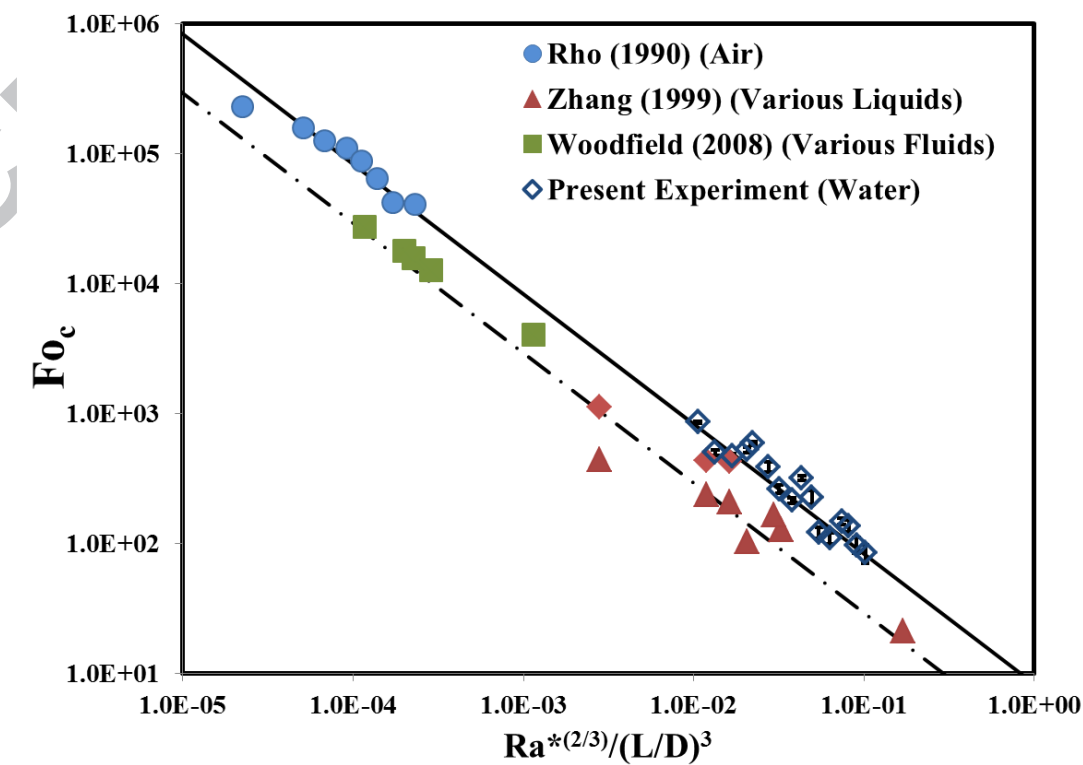

Figure 3 Comparison of the present experimental results for water with the correlations of Zhang et al [19] 
By examining the temperature-rise data it is apparent that the time of onset of natural convection corresponds reasonably well to the 'knee' of the curve in the temperature rise data illustrated by the intersection of straight lines in Fig. 2. The horizontal line represents steadystate free convection for the wire and the straight line passing through the linear section of the data corresponds to conduction only. Since the two regimes can be defined by equations representing Nusselt numbers for conduction and convection, the intersection point is the time when the Nusselt number for convection becomes equal to the Nusselt number of transient conduction only. Therefore, we propose that a reasonable estimate for the critical time for the onset of convection effects can be obtained by simultaneously solving relations that predict the Nusselt numbers for the two regimes:

$$
\mathrm{Nu} \text { Conduction only }=\mathrm{Nu} \text { Convection dominated }
$$

The temperature rise of the hotwire for the initial linear region (conduction only - see Figure 2) has been given by Carslaw and Jaeger [42]:

$$
\Delta T=\frac{q^{\prime}}{4 \pi k} \ln \left(\frac{4 \alpha t}{r_{w i r e}{ }^{2} \xi}\right)
$$

where $q^{\prime}$ is the heat generation per unit length of the wire, $k$ the thermal conductivity of the fluid, $\alpha$ thermal diffusivity of fluid and $\xi=1.781$ is the exponential of Euler's constant. By substituting Eq. (9) and Eq. (13) into Eq. (10), the Nusselt number for conduction from a long wire into a surrounding fluid is given as:

$$
\mathrm{Nu}_{\mathrm{L}}=\frac{4 \mathrm{~L}}{\mathrm{D} \ln \left(\frac{4 \mathrm{Fo}}{\xi}\right)}
$$

Concerning the horizontal line in Figure 2, a number of equations for the Nusselt number of free convection around a vertical thin cylinder are reported in the literature [48]. One of them is the theoretically derived equation by Le Fevre and Ede [49]:

$$
\overline{\mathrm{Nu}}=\frac{4}{3}\left[\frac{7 \mathrm{Gr} \cdot \mathrm{Pr}}{5(20+21 \mathrm{Pr})}\right]^{\frac{1}{4}}+\frac{4(272+315 \mathrm{Pr}) \mathrm{L}}{35(64+63 \mathrm{Pr}) \mathrm{D}}
$$

Another equation for the vertically heated cylinders is the empirical correlation proposed by Yang [50]:

$$
N u_{L}^{0.5}=0.6\left(\frac{L}{D}\right)^{0.5}+0.387\left[\frac{R a}{\left(1+\left(\frac{0.492}{P r}\right)^{\frac{9}{16}}\right)^{\frac{16}{9}}}\right]^{\frac{1}{6}}
$$

As shown in Figure 4, the data for natural convection in DIW from this work correlates with:

$$
N u=50 R a^{0.13}
$$


Thus, substituting Eq. (14) and Eq. (15),(16) or (17) into Eq. (12) gives a means of estimating the critical Fourier number $F o_{c}$ (or time) for onset of free convection. A similar approach can also give an estimate for the time of onset of thermomagnetic convection.

In order to apply Eq. (12) to predicting the onset of thermomagnetic convection, a correlation for the thermomagnetic convection-dominated Nusselt number in Ferrofluid is required. For this purpose (as illustrated in Figure 4) the present results for ferrofluid suggest that the Nusselt number for thermomagnetic convection-dominated heat transfer from the wire can be correlated with

$$
N u=1.23 R a_{m}^{0.23}
$$

Eq. (18) shows that the Nusselt number for thermomagnetic convection of the ferrofluid is proportional to magnetic Rayleigh number with a power of approximately one quarter, which is a common proportionality for the natural convection in the literature [48, 51-54]. For determining the Nusselt number for the ferrofluid in Figure 4, the local minimum in the curve shown in Figure 2 (at time $\approx 1.5 \mathrm{~s}$ ) was used.

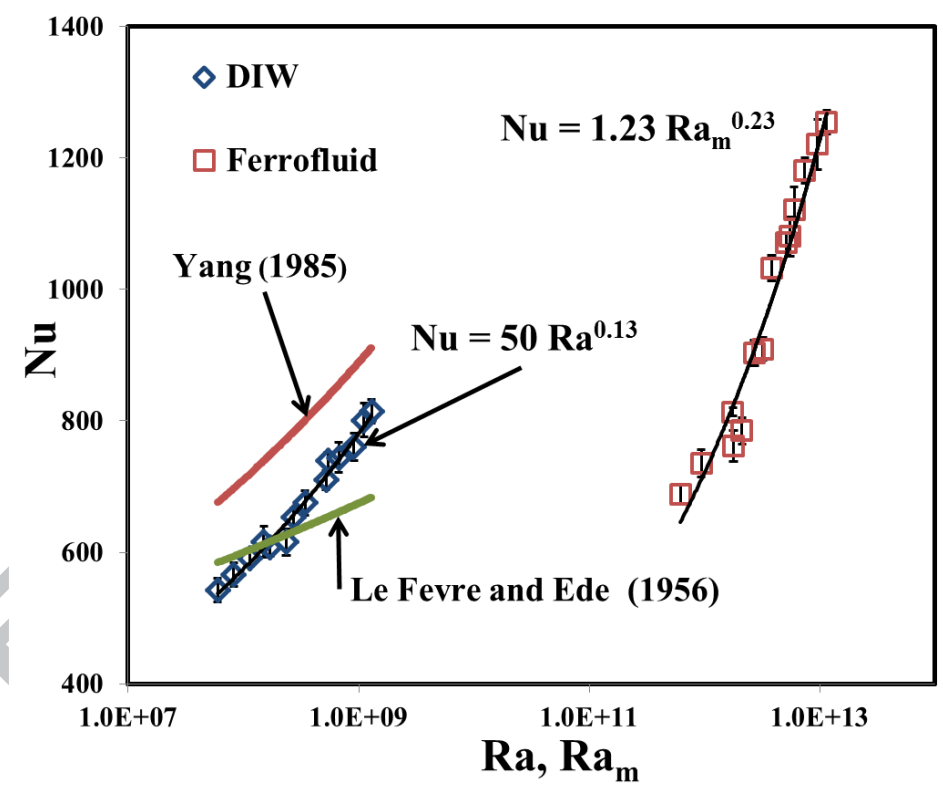

Figure 4 Variation of the $\mathrm{Nu}_{\mathrm{L}}$ with $\mathrm{Ra}$ and $\mathrm{Ra}_{\mathrm{m}}$ for $\mathrm{DIW}$ and Ferrofluid, respectively.

By substituting the correlations for the Nusselt number as a function of Rayleigh number in the form of $N u_{\text {convection }}=X R a^{Y}$ (both for free convection and thermomagnetic convection) in the equality of Nusselt numbers, an equation for predicting $F o_{c}$ can be derived as:

$$
\ln F o_{c}=\frac{4}{X} R a^{-Y}+\ln \frac{\xi}{4}
$$

Where $X=50, Y=0.13$ for free convection and $X=1.22, Y=0.23$ for thermomagnetic convection. Figure 5 shows that relatively good agreement is observed between measured and predicted $\mathrm{Fo}_{c}$ for both free convection of DIW and thermomagnetic convection of ferrofluid. It is also observed, by point by point comparison of the results for DIW and ferrofluid, that although 
$\mathrm{Fo}_{c}$ for both cases decreases by increasing the applied current, this decrease is more substantial for ferrofluid.

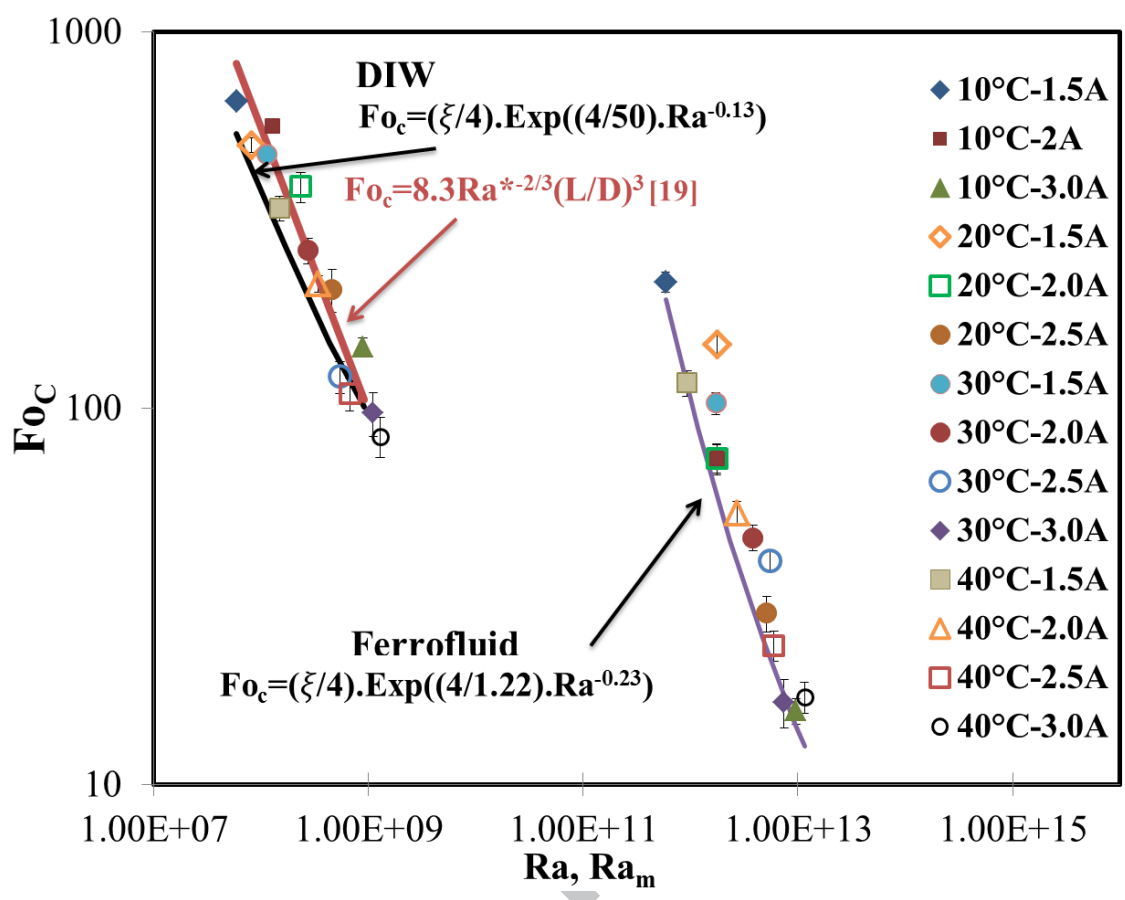

Figure 5 Comparison of the experimentally determined critical Fourier number for the onset of convection with the predictions for DIW and Ferrofluid (Eq. 19).

\section{Interpretation and definitions of dimensionless groups}

The present study relies heavily on the use of dimensionless groups and analogies between natural convection and thermomagnetic convection. The analogy is justifiable because both natural convection and thermomagnetic convection introduce a body force term to the Navier-Stokes equation which is driven by temperature gradients. For example, in Eq. (7) the gravitational body force term (fourth term on the right) is often replaced by $g \beta \Delta T$ which is known as the Boussinesq approximation for natural convection. Thus (as a first approximation), both terms are proportional to a temperature change with respect to a reference condition even though the spatial direction of the two terms is different (here radial and axial for thermomagnetic and natural convection, respectively).

The magnetic Rayleigh number has been defined in several studies [7, 55-58] with similar physical interpretations but with different magnetic field configurations. In these prior studies, the magnetic field is imposed externally with a single direction [56, 57] or dipole [7] arrangement. In this study, however, the magnetic field is that of a single current-carrying wire and as a result, the magnetic Rayleigh number (Eq. (8)) involves a radial space dimensions. Moreover, instead of expressing the magnetic Rayleigh number in terms of a pyromagnetic constant and general magnetic field gradients $[44,59]$ we have used a more 
convenient form for evaluation in terms of the electrical current in the wire. Therefore, the Rayleigh number given by Eq. (8) should only be used for circumstances where the field is generated by an electric current in a single conductor. The interpretation of the present magnetic Rayleigh number is the ratio of thermomagnetic body forces from a single electrical conductor to viscous body forces multiplied by the ratio of viscous to thermal diffusion.

A further insightful dimensionless consideration is the ratio of $\mathrm{Ra}_{\mathrm{m}}$ and $\mathrm{Ra}$. It is evident in Figure 4 that the magnetic Rayleigh number is few orders of magnitude larger than conventional Rayleigh number. The ratio of these two dimensionless numbers is the ratio of magnetic to gravitational buoyancy forces. This large ratio $(O E+O 3)$ is consistent with the earlier occurrence of thermomagnetic convection rather than natural convection.

Our proposed criterion for onset of thermomagnetic convection is that the ratio of a transient conduction dominated Nusselt number to a steady-state convection dominated Nusselt number is unity (Eq. (12)). Usually, Nusselt numbers are interpreted as the ratio of convection heat transfer to conduction heat transfer. This is certainly the case for $\mathrm{Nu}_{\text {convection }}$

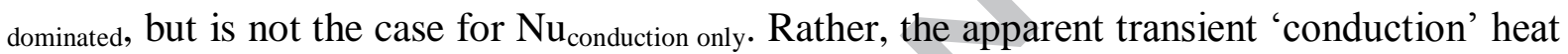
transfer coefficient from Eq. (14) arises from applying Newton's law of cooling (Eq. (9)) in the limit of conduction-dominated heat transfer. (Note that it is quite common to apply Newton's law of cooling to conduction heat transfer situations e.g. - when defining overall heat transfer coefficients in heat exchangers). Therefore $\mathrm{Nu}_{\text {conduction dominated }}$ can be interpreted as the ratio of thermal resistance due to conduction in the axial direction of the domain to the apparent transient thermal resistance between a transiently heated cylinder and an infinite fluid. The proposed onset criterion has justification in that we are looking for a boundary between the two regimes - namely conduction-dominated and thermomagnetic convectiondominated heat transfer for the wire.

\section{Conclusion}

The onset of thermomagnetic convection of ferrofluid around a vertical heated wire in a thermal conductivity measurement cell, for the first time, was experimentally studied and compared with the onset of free convection for DIW. Experimental results for DIW were validated by comparison with the available correlations in the literature. Nusselt number of the flow has been correlated as a function of magnetic Rayleigh number for thermomagnetic convection. Equations for predicting the onset of both thermomagnetic convection and free convection were proposed. Thermomagnetic convection occurs due to temperature gradients affecting of a ferrofluid in a magnetic field. It was shown that generally, thermomagnetic convection happens earlier than free convection. The time for the onset of thermomagnetic convection changes exponentially with the increase in the current supplied to the wire. Since Fo increases with decreasing length scale, thermomagnetic convection can be used for microscale applications where immediate cooling is required and free convection is not effective. The effect of varying radius of the wire as the characteristic length the onset of convection needs to be studied in future. Also the effectiveness of thermomagnetic convection for temperatures above the boiling point of the liquid is yet to be examined. 


\section{Acknowledgment}

This work was performed in part at the Queensland Node of the Australian National Fabrication Facility, a company established under the National Collaborative Research Infrastructure Strategy to provide nano- and microfabrication facilities for Australia's researchers. The authors acknowledge funding support from Australian Research Council through the ARC linkage grant (LP150100153).

\section{Nomenclature}

$\vec{B}$

Flux density, $\frac{N \cdot m}{A}$

$\mathrm{D}$

Diameter of wire, $m$

f

Body force

$\vec{H}$

Magnetic field strength, $\frac{A}{m}$

I

Current, $A$

$k_{B}$

Boltzmann's constant

$\mathrm{k}$

Thermal conductivity, $\frac{w}{m \cdot k}$

L

Length of wire, $m$

$\vec{M}$

Magnetization, $\frac{A}{m}$

$\mathrm{R}$

Resistance of wire, $\mathrm{Ohm}$

Radius, $m$

$\mathrm{t}$

Time, $s$

\section{Greek Letters}

Magnetic susceptibility

$\mu_{0}$

Magnetic permeability of free space, $\frac{N}{A^{2}}$

$\alpha$

Thermal diffusivity, $\frac{m^{2}}{s}$

$\eta$

Dynamic viscosity, $\frac{N . s}{m^{2}}$

$v$

Kinematic viscosity, $\frac{m^{2}}{s}$ 
$\beta \quad$ Thermal expansion coefficient, $\frac{1}{K}$

Non-dimensional numbers

$\begin{array}{ll}\mathrm{Nu} & \text { Nusselt number } \\ \mathrm{Ra} & \text { Rayleigh number } \\ \text { Subscripts } & \\ \mathrm{I} & \text { Initial value } \\ 0 & \text { Reference value } \\ \mathrm{C} & \text { Critical value }\end{array}$

\section{References}

[1] M. Zahn, Magnetic fluid and nanoparticle applications to nanotechnology, Journal of nanoparticle research, 3 (2001) 73-78.

[2] C. Scherer, A.M. Figueiredo Neto, Ferrofluids: properties and applications, Brazilian Journal of Physics, 35 (2005) 718-727.

[3] K. Raj, R. Moskowitz, Commercial applications of ferrofluids, Journal of Magnetism and Magnetic Materials, 85 (1990) 233-245.

[4] P. Berger, N.B. Adelman, K.J. Beckman, D.J. Campbell, A.B. Ellis, G.C. Lisensky, Preparation and properties of an aqueous ferrofluid, J. Chem. Educ, 76 (1999) 943.

[5] J.-C. Bacri, R. Perzynski, D. Salin, V. Cabuil, R. Massart, Magnetic colloidal properties of ionic ferrofluids, Journal of Magnetism and Magnetic Materials, 62 (1986) 36-46.

[6] N.-T. Nguyen, Micro-magnetofluidics: interactions between magnetism and fluid flow on the microscale, Microfluidics and nanofluidics, 12 (2012) 1-16.

[7] A. Mukhopadhyay, R. Ganguly, S. Sen, I.K. Puri, A scaling analysis to characterize thermomagnetic convection, International Journal of Heat and Mass Transfer, 48 (2005) 3485-3492.

[8] A. Bozhko, G. Putin, Thermomagnetic convection as a tool for heat and mass transfer control in nanosize materials under microgravity conditions, Microgravity Science and Technology, 21 (2009) 89-93.

[9] M. Krakov, I. Nikiforov, To the influence of uniform magnetic field on thermomagnetic convection in square cavity, Journal of Magnetism and Magnetic Materials, 252 (2002) 209-211.

[10] A. Vatani, P.L. Woodfield, N.-T. Nguyen, D.V. Dao, Thermomagnetic convection around a current-carrying wire in ferrofluid, Journal of Heat Transfer, (2017).

[11] D. Yadav, Numerical solution of the onset of natural convection in a rotating nanofluid layer induced by purely internal heating, International Journal of Applied and Computational Mathematics, (2017) 1-19.

[12] D. Yadav, D. Lee, H.-H. Cho, J. Lee, The onset of double-diffusive nanofluid convection in a rotating porous medium layer with thermal conductivity and viscosity variation: a revised model, Journal of Porous media, 19 (2016). 
[13] D. Yadav, R. Mohamed, H.H. Cho, J. Lee, Effect of Hall Current on the Onset of MHD Convection in a Porous Medium Layer Saturated by a Nanofluid, Journal of Applied Fluid Mechanics, 9 (2016).

[14] S. Sarkar, S. Ganguly, G. Biswas, Buoyancy driven convection of nanofluids in an infinitely long channel under the effect of a magnetic field, International Journal of Heat and Mass Transfer, 71 (2014) 328-340.

[15] H.M. Roder, R.A. Perkins, A. Laesecke, C.A.N. de Castro, Absolute steady-state thermal conductivity measurements by use of a transient hot-wire system, Journal of research of the National Institute of Standards and Technology, 105 (2000) 221.

[16] A. Davaille, C. Jaupart, Onset of thermal convection in fluids with temperature-dependent viscosity: Application to the oceanic mantle, Journal of Geophysical Research: Solid Earth, 99 (1994) 19853-19866.

[17] J.H.L. S.T. Ro, J.Y. Yoo, Onset of natural convection effect in transient hot-wire system, Thermal conductivity 21, Edited by Cremers and Fine, Plenum Press, (1990) 151-163.

[18] J.F. P.L. Woodfield, M. Fujii, Y. Takata, K. Shinzato, Numerical Simulation of Natural Convection in a Transient Short-Hot-Wire Thermal Conductivity Cell, 熱物性, 22 (2008) 217-222.

[19] X. Zhang, S. Fujiwara, Z. Qi, M. Fujii, Natural convection effect on transient short-hot-wire method, JASMA: Journal of the Japan Society of Microgravity Application, 16 (1999) 129-135.

[20] D. Yadav, J. Lee, The onset of MHD nanofluid convection with Hall current effect, The European Physical Journal Plus, 130 (2015) 162.

[21] D. Yadav, C. Kim, J. Lee, H.H. Cho, Influence of magnetic field on the onset of nanofluid convection induced by purely internal heating, Computers \& Fluids, 121 (2015) 26-36.

[22] D. Yadav, J. Wang, R. Bhargava, J. Lee, H.H. Cho, Numerical investigation of the effect of magnetic field on the onset of nanofluid convection, Applied Thermal Engineering, 103 (2016) 14411449.

[23] S. Chandrasekhar, Hydrodynamic and hydromagnetic stability, Courier Corporation, 2013.

[24] S. Sarkar, S. Ganguly, Fully developed thermal transport in combined pressure and electroosmotically driven flow of nanofluid in a microchannel under the effect of a magnetic field, Microfluidics and Nanofluidics, 18 (2015) 623-636.

[25] S. Sarkar, S. Ganguly, P. Dutta, Electrokinetically induced thermofluidic transport of power-law fluids under the influence of superimposed magnetic field, Chemical Engineering Science, (2017).

[26] S. Ganguly, S. Sarkar, T.K. Hota, M. Mishra, Thermally developing combined electroosmotic and pressure-driven flow of nanofluids in a microchannel under the effect of magnetic field, Chemical Engineering Science, 126 (2015) 10-21.

[27] S. Sarkar, S. Ganguly, P. Dutta, Thermofluidic characteristics of combined electroosmotic and pressure driven flows in narrow confinements in presence of spatially non-uniform magnetic field, International Journal of Heat and Mass Transfer, 104 (2017) 1325-1340.

[28] G. Pacitto, C. Flament, J.-C. Bacri, M. Widom, Rayleigh-Taylor instability with magnetic fluids: Experiment and theory, Physical Review E, 62 (2000) 7941.

[29] A.A. Bozhko, Onset of convection in magnetic fluids, Physics Procedia, 9 (2010) 176-180.

[30] H. Engler, S. Odenbach, Influence of parametric modulation on the onset of thermomagnetic convection, PAMM, 9 (2009) 515-516.

[31] H. Engler, S. Odenbach, Thermomagnetic convection in magnetic fluids influenced by a time-modulated magnetic field, PAMM, 8 (2008) 10951-10952.

[32] T. Tynjälä, J. Ritvanen, Simulations of thermo-magnetic convection in an annulus between two concentric cylinders, (2004).

[33] H. Engler, A. Lange, D. Borin, S. Odenbach, Hindrance of thermomagnetic convection by the magnetoviscous effect, International Journal of Heat and Mass Transfer, 60 (2013) 499-504.

[34] A. Lange, Thermomagnetic convection of magnetic fluids in a cylindrical geometry, Physics of Fluids, 14 (2002) 2059-2064.

[35] M.I. Shliomis, B.L. Smorodin, S. Kamiyama, The onset of thermomagnetic convection in stratified ferrofluids, Philosophical Magazine, 83 (2003) 2139-2153. 
[36] C. Nanjundappa, I. Shivakumara, H. Prakash, Effect of Coriolis force on thermomagnetic convection in a ferrofluid saturating porous medium: A weakly nonlinear stability analysis, Journal of Magnetism and Magnetic Materials, 370 (2014) 140-149.

[37] R. Sekar, K. Raju, R. Vasanthakumari, A linear analytical study of Soret-driven ferrothermohaline convection in an anisotropic porous medium, Journal of Magnetism and Magnetic materials, 331 (2013) 122-128.

[38] C. Nanjundappa, H. Prakash, I. Shivakumara, J. Lee, Effect of temperature dependent viscosity on the onset of Bénard-Marangoni ferroconvection, International Communications in Heat and Mass Transfer, 51 (2014) 25-30.

[39] A. Gear, T.J. Rundle, J. Barhite, J. Rice, Onset of Normal Field Instability in a Ferrofluid in Microgravity, in: Proceedings of the Wisconsin Space Conference, 2016.

[40] S. Saravanan, H. Yamaguchi, Onset of centrifugal convection in a magnetic-fluid-saturated porous medium, Physics of Fluids, 17 (2005) 084105.

[41] R. Rusconi, W.C. Williams, J. Buongiorno, R. Piazza, L.-W. Hu, Numerical analysis of convective instabilities in a transient short-hot-wire setup for measurement of liquid thermal conductivity, International Journal of Thermophysics, 28 (2007) 1131-1146.

[42] H.S. Carslaw, J.C. Jaeger, Conduction of heat in solids, Clarendon Press Oxford, 1959.

[43] H.M. Roder, A. Richard, Absolute Steady-State Thermal Conductivity Measurements by Use of a Transient, Journal of Research of the National Institute of Standards and Technology, 105 (2000).

[44] B. Finlayson, Convective instability of ferromagnetic fluids, Journal of Fluid Mechanics, 40 (1970) 753-767.

[45] T.L. Bergman, F.P. Incropera, D.P. DeWitt, A.S. Lavine, Fundamentals of heat and mass transfer, John Wiley \& Sons, 2011.

[46] A. Vatani, P.L. Woodfield, D.V. Dao, A miniaturized transient hot-wire device for measuring thermal conductivity of non-conductive fluids, Microsystem Technologies, 22 (2016) 2463-2466.

[47] M. Corcione, Empirical correlating equations for predicting the effective thermal conductivity and dynamic viscosity of nanofluids, Energy Conversion and Management, 52 (2011) 789-793.

[48] C.O. Popiel, Free convection heat transfer from vertical slender cylinders: a review, Heat Transfer Engineering, 29 (2008) 521-536.

[49] E. Le Fevre, A. Ede, Laminar free convection from the outer surface of a vertical circular cylinder, in: Proceedings of the 9th International Congress Applied Mechanics, 1956, pp. 175-183.

[50] S. Yang, General correlating equations for free convection heat transfer from a vertical cylinder, in: Proceedings of the International Symposium on Heat Transfer, Hemisphere Publ. Corp., Peking, 1985, pp. 153-159.

[51] M. Jakob, W. Linke, Der Wärmeübergang beim Verdampfen von Flüssigkeiten an senkrechten und waagerechten Flächen, Phys. Z, 36 (1935) 267-280.

[52] W. King, The basic laws and data of heat transmission, American Society of Mechanical Engineers, 1932.

[53] V.T. Morgan, The overall convective heat transfer from smooth circular cylinders, Advances in heat transfer, 11 (1975) 199-264.

[54] W. Nusselt, Die Wärmeübgabe eines waagrecht liegenden Drahtes oder Rohres in Flüssigkeiten und gasen, Ver Deut Ing, 73 (1929) 1475-1478.

[55] H. Rahman, S.A. Suslov, Thermomagnetic convection in a layer of ferrofluid placed in a uniform oblique external magnetic field, Journal of Fluid Mechanics, 764 (2015) 316-348.

[56] T. Tynjälä, A. Hajiloo, W. Polashenski, P. Zamankhan, Magnetodissipation in ferrofluids, Journal of Magnetism and Magnetic Materials, 252 (2002) 123-125.

[57] S. Odenbach, Microgravity experiments on thermomagnetic convection in magnetic fluids, Journal of magnetism and magnetic materials, 149 (1995) 155-157.

[58] D. Zablotsky, A. Mezulis, E. Blums, Surface cooling based on the thermomagnetic convection: numerical simulation and experiment, International Journal of Heat and Mass Transfer, 52 (2009) 5302-5308. 
[59] A. Bozhko, G. Putin, Magnetic action on convection and heat transfer in ferrofluid, (2004).

\section{Highlights}

- Onset of thermomagnetic convection around a vertically oriented heated micro-wire was analytically and experimentally studied

- The time for the onset of natural convection was validated against existing correlations in the literature.

- The time corresponding to the onset of thermomagnetic convection was characterized as critical Fourier number and an equation was proposed for that as a function of magnetic Rayleigh number.

- Suggestions for future work are presented. 


\section{Accepted Manuscript}

Research articles

Onset of thermomagnetic convection around a vertically oriented hot-wire in ferrofluid

Ashkan Vatani, Peter Lloyd Woodfield, Nam-Trung Nguyen, Dzung Viet Dao

PII: S0304-8853(17)31517-2

DOI: https://doi.org/10.1016/j.jmmm.2018.02.040

Reference: MAGMA 63717

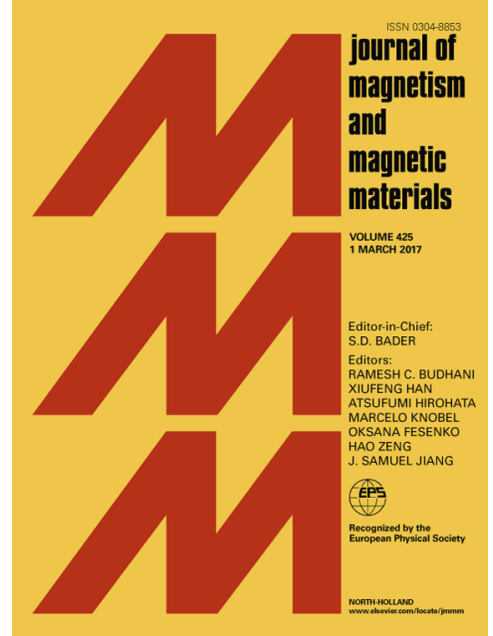

To appear in:

Journal of Magnetism and Magnetic Materials

Received Date:

16 May 2017

Revised Date:

27 December 2017

Accepted Date:

12 February 2018

Please cite this article as: A. Vatani, P.L. Woodfield, N-T. Nguyen, D.V. Dao, Onset of thermomagnetic convection around a vertically oriented hot-wire in ferrofluid, Journal of Magnetism and Magnetic Materials (2018), doi: https://doi.org/10.1016/j.jmmm.2018.02.040

This is a PDF file of an unedited manuscript that has been accepted for publication. As a service to our customers we are providing this early version of the manuscript. The manuscript will undergo copyediting, typesetting, and review of the resulting proof before it is published in its final form. Please note that during the production process errors may be discovered which could affect the content, and all legal disclaimers that apply to the journal pertain. 